\title{
Exploring the chicken embryo as a possible model for studying Listeria monocytogenes pathogenicity
}

\author{
Jonas Gripenland ${ }^{1,2,3 t \neq}$, Christopher Andersson ${ }^{1,2,3 \neq}$ and Jörgen Johansson ${ }^{1,2,3 *}$ \\ ' Department of Molecular Biology, Umeå University, Umeå, Sweden \\ ${ }^{2}$ Laboratory for Molecular Infection Medicine Sweden, Umeå University, Umeå, Sweden \\ ${ }^{3}$ Umeå Centre for Microbial Research, Umeå University, Umeå, Sweden
}

Edited by:

Stephanie M. Seveau, The Ohio

State University, USA

Reviewed by:

Sarah E. F. D'Orazio, University of

Kentucky, USA

Oscar Gilberto Gomez-Duarte,

Vanderbilt University School of

Medicine, USA

\section{*Correspondence:}

Jörgen Johansson, Department of

Molecular Biology, Umeå University,

Försöriningsvägen 1, 90187 Umeå,

Sweden

e-mail:jorgen.johansson@

molbiol.umu.se

${ }^{\dagger}$ Present address:

Jonas Gripenland, Division of

Neurology, Department of Clinical

Neuroscience, Karolinska University

Hospital, Stockholm, Sweden

¥ These authors have contributed

equally to this work.
Listeria monocytogenes is a bacterial pathogen capable of causing severe infections in humans, often with fatal outcomes. Many different animal models exist to study L. monocytogenes pathogenicity, and we have investigated the chicken embryo as an infection model: What are the benefits and possible drawbacks? We have compared a defined wild-type strain with its isogenic strains lacking well-characterized virulence factors. Our results show that wild-type L. monocytogenes, already at a relatively low infection dose $\left(\sim 5 \times 10^{2} \mathrm{cfu}\right)$, caused death of the chicken embryo within $36 \mathrm{~h}$, in contrast to strains lacking the main transcriptional activator of virulence, PrfA, or the cytolysin LLO. Surprisingly, strains lacking the major adhesins InIA and InIB caused similar mortality as the wild-type strain. In conclusion, our results suggest that the chicken embryo is a practical model to study L. monocytogenes infections, especially when analyzing alternative virulence pathways independent of the $\operatorname{In} \mid \mathrm{A}$ and $\operatorname{In} \mid \mathrm{B}$ adhesins. However, the route of infection might be different from a human infection. The chicken embryo model and other Listeria infection models are discussed.

Keywords: Listeria monocytogenes, chicken embryo, PrfA, virulence: InIA, LLO, InIB

\section{INTRODUCTION}

Listeria monocytogenes is a Gram-positive human bacterial pathogen, capable of causing severe infections, Listeriosis, predominantly in immuno-compromised patients. Once ingested, the bacteria can cross the intestinal barrier and disseminate via the blood and lymph system to the liver and spleen, the primary sites for replication of L. monocytogenes (Vazquez-Boland et al., 2001; Lecuit, 2007). The route of infection follows a spatiotemporal pattern: In healthy hosts, the bacteria replicates for less than a week before being cleared by the immune system (VazquezBoland et al., 2001; Lecuit, 2007). In immuno-compromised patients, the bacterium continue to replicate and eventually disseminate into the blood-stream where it can cause septicemia. The bacteria may subsequently also pass the blood-brain barrier, leading to meningo-encephalitis or meningitis (reviewed in Vazquez-Boland et al., 2001; Lecuit, 2007). If the host is pregnant, the bacterium is capable of crossing the materno-fetal barrier resulting in abortion or severe neonatal infections. The latter phases of a Listeria infection have a mortality incidence between 20 and $30 \%$ (Vazquez-Boland et al., 2001; Lecuit, 2007). To cross the intestinal barrier, L. monocytogenes uses an adhesin, InlA, which recognizes E-cadherin (Mengaud et al., 1996). Translocation through the placental barrier requires the concerted action of InlA and another adhesin, InlB (Disson et al., 2008), where the latter primarily recognizes the c-Met receptor
(Shen et al., 2000). The molecular mechanism underlying bloodbrain passage has not yet been resolved. Other proteins involved in cellular entry have also been recognized (i.e., Vip and Auto, Cabanes et al., 2004, 2005). Once internalized, Listeria is able to escape the phagosome through the action of Listeriolysin $\mathrm{O}$ (LLO), before it spreads from cell to cell by polymerizing actin in an ActA-dependent mechanism (Cossart, 2011). Almost all virulence factors are controlled by one transcriptional activator, PrfA, which bind PrfA consensus binding sites located in the promoter region of PrfA regulated genes, thus activating their expression (Freitag et al., 2009; de las Heras et al., 2011). Expression of PrfA is controlled at several different layers: At the transcriptional level through different promoters, at the post-transcriptional level by both a thermosensor lying in the $5^{\prime}$-untranslated RNA and by the action of small regulatory RNAs (Freitag et al., 2009; Gripenland et al., 2010). Finally, the activity of PrfA is believed to be controlled at the post-translational level, through a hitherto unknown PrfA binding factor (de las Heras et al., 2011).

\section{ANIMAL MODEL SYSTEMS FOR STUDYING LISTERIA PATHOGENICITY}

Using cultured cell-lines, a tremendous knowledge of the different bacterial factors and cellular events that Listeria master has been gained. These cells are generally transformed cell-lines with different origins (cell-type and species), allowing a large choice for 
analyzing Listeria infection. To further understand the molecular mechanisms underlying Listeria infection, several animal models have been utilized. Accumulating in vivo animal data suggest that the mechanism of Listeria infection might be much more complex than previously anticipated, extrapolating from in vitro cell-culture data (Disson and Lecuit, 2013).

The mice (Mus musculus) model is frequently used to study a Listeria infection in a laboratory setting (Lecuit, 2007). The advantages are obvious; mice are relatively easy and inexpensive to maintain, the genome has been sequenced and a plethora of knock-out lineages are available for host studies. However, a drawback of using mice as a model for Listeria infections is the non-functional interaction between mouse E-cadherin and InlA, due to a glutamate instead of proline at position 16 of E-cadherin (Lecuit et al., 1999). This drawback has been resolved by replacing the glutamate to proline in position 16 of E-cadherin in mice (Lecuit et al., 2001; Disson et al., 2008) but also by "murinizing" InlA for it to recognize mice E-cadherin (Wollert et al., 2007).

Another model that has been used to study Listeria infectivity, albeit at a lower frequency, is the guinea pig (Cavia porcellus). Unlike mice, guinea pigs carry an E-cadherin recognizing InlA, which allows for an efficient translocation through the intestinal barrier (Lecuit et al., 2001; Khelef et al., 2006). However, the guinea pig was unable to provide a successful InlB:c-Met interaction, making this model system less suitable for studying the infection process downstream of intestinal crossing (Khelef et al., 2006).

Gerbil (Meriones unguiculates) which is a natural host for Listeria monocytogenes, allow InlA and InlB to successfully bind E-cadherin and c-Met, respectively (Disson et al., 2008). Therefore, the gerbil has been suggested as the animal of choice when studying Listeria infections. There are however drawbacks when using the gerbil model: The genome sequence of gerbils has not yet been published and they are not yet as commercially available as mice. Also, despite having functional InlA and InlB pathways, it was recently shown that very high doses of Listeria $\left(\sim 1 \times 10^{9}\right)$ were required to cause stillbirth in gerbils (Roulo et al., 2014).

The Rhesus monkey (Macaca mulatta), a non-human primate, has also been used to study Listeria pathogenicity. Its genome has been sequenced, the infection route is believed to closely mimic the human route and similar doses of Listeria cause stillbirth in both species (Smith et al., 2008). However, the Rhesus monkey model is expensive, requires large facilities and may be more ethically demanding.

Other animal infection models of L. monocytogenes include the zebra fish (Danio rerio), Drosophila melanogaster and Caenorhabditis elegans (Mansfield et al., 2003; Thomsen et al., 2006; Levraud et al., 2009). Their genomes have all been sequenced, they are inexpensive and optically accessible. Yet, since all three models are cold-blooded with a temperature maximum of $30^{\circ} \mathrm{C}$, appropriate virulence factor expression might not reflect a human infection, which lies at $37^{\circ} \mathrm{C}$.

Birds are natural hosts for L. monocytogenes and the bacterium has been associated with outbreaks in chicken broilers (Cooper et al., 1992; Vazquez-Boland et al., 2001). A possible avian model of Listeria infections is represented by chicken embryos (Gallus gallus). The chicken model has been used to assess the virulence of several pathogens, like: Clostridium perfringens, Staphylococcus aureus, Escherichia coli, Salmonella enteridis, and Francisella tularensis (Wang et al., 2008; Horzempa et al., 2010; Oh et al., 2012; Polakowska et al., 2012; Alnassan et al., 2013). Many studies have also investigated the pathogenic potential of clinically and environmentally isolated L. monocytogenes using chicken embryos (Terplan and Steinmeyer, 1989; Buncic and Avery, 1996; Norrung and Andersen, 2000; Olier et al., 2002, 2003; Severino et al., 2007; Yin et al., 2011). These reports clearly indicate that chicken embryos can be used as a model for Listeria infection, although the molecular mechanism causing the infection has not been examined in detail. The pore-forming cytolysin LLO have previously been shown to be important for chicken embryo infection (Jiang et al., 2005), but the impact of other PrfA-regulated factors have not been assessed.

\section{USING ISOGENIC L. MONOCYTOGENES STRAINS TO INFECT CHICKEN EMBRYOS}

In order to examine the plausibility of using the chicken embryo model to assess Listeria pathogenicity as well as analyzing the importance of the PrfA virulence regulon, the EGDe (wild-type) strain and its isogenic $\triangle p r f A$ strain were used (see Supplementary Materials for methods). Infecting eggs with $5 \times 10^{2}$ wild-type bacteria lead to death of all eggs within $48 \mathrm{~h}$ (Figure 1A). Similar amounts of Listeria wild-type bacteria have been used in previous studies when infecting chicken embryos (Norrung and Andersen, 2000; Olier et al., 2002, 2003; Severino et al., 2007; Yin et al., 2011). Using the same amount of the $\Delta$ prfA strain, only $20 \%$ of the chicken embryos were killed $72 \mathrm{~h}$ post-infection (Figure 1A). The inability of the $\triangle p r f A$ strain to cause death of chicken embryos prompted us to investigate whether this was accompanied by a decreased infection (i.e. a reduced bacterial growth within the embryo). We therefore isolated livers from infected living embryos at $34 \mathrm{~h}$ post-infection. The wild-type infected embryos showed almost a 100-fold higher bacterial count compared with the $\triangle p r f A$ strain in the liver (Figure 1B). We did not detect any obvious difference in the weight and appearance of the livers isolated from wild-type and $\triangle p r f A$ strains (data not shown).

To further examine the PrfA pathway and in more detail pin-point the role of certain PrfA-regulated virulence factors, isogenic mutant strains of $h l y$; inlA, or inlB were used in a chicken embryo survival experiment together with the wildtype strain. The pore-forming cytolysin Listeriolysin O (LLO, encoded by $h l y$ ) was required for a successful Listeria infection (Figure 2). This is in agreement with previous studies (McKay and Lu, 1991; Jiang et al., 2005), and highlights the importance of LLO during infection. Listeria infection of several epithelial cells require a successful adhesin: receptor interaction (Gaillard et al., 1991). The best characterized adhesins in Listeria are InlA, which recognizes E-cadherin, and InlB, which recognizes the c-Met receptor (Mengaud et al., 1996; Shen et al., 2000). Surprisingly, absence of either InlA or InlB did not attenuate Listeria-mediated killing of chicken embryos as compared with the wild-type (Figure 2). 

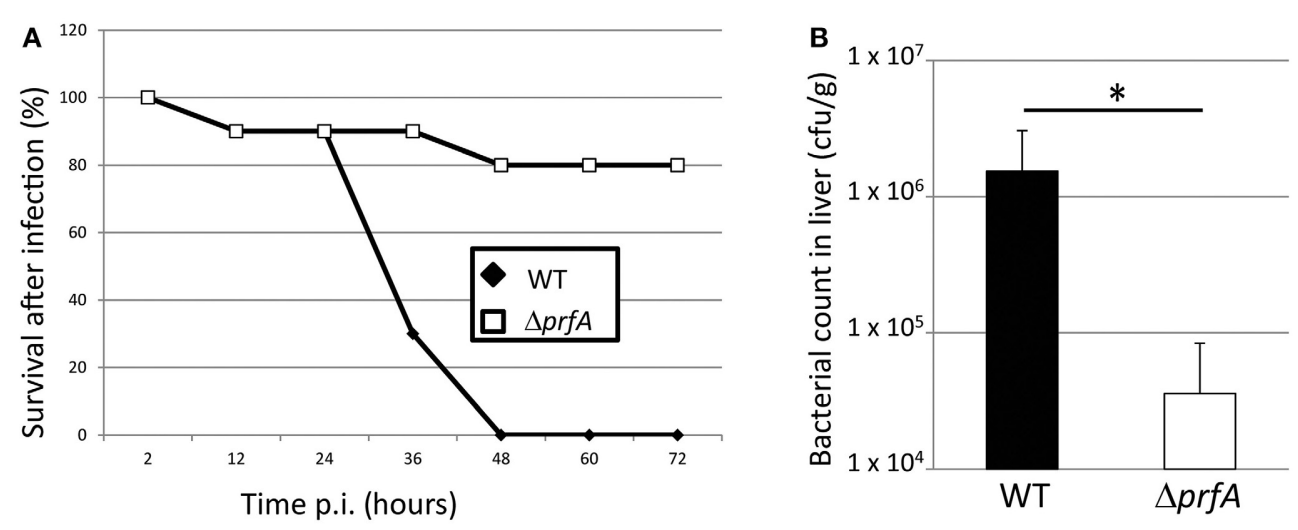

FIGURE 1 | (A) Survival curve of chicken embryos infected with L. monocytogenes wild-type (EGDe) and the isogenic $\Delta$ prfA strain. $\sim 5 \times 10^{2}$ bacteria were inoculated into 9-day old chicken embryos, which were followed for $72 \mathrm{~h}$ by light candling. Death are shown as mean of 5 experiments ( $n=17$ for the WT strain and $n=16$ for the $\Delta$ prfA strain). (B) Bacterial counts of wild-type and $\triangle p r f A$ strains in the liver of chicken embryos. $\sim 5 \times 10^{2}$ bacteria were inoculated into 9-day old chicken embryos that were sacrificed after $34 \mathrm{~h}$. The liver was isolated from living embyos and the number of viable bacteria was determined ( $n=6$ for the WT strain and $n=8$ for the $\triangle$ prfA strain, divided over 3 experiments). Error bars show standard error. The difference is statistically significant $(p<0.05$ through students $T$-test) and marked with an asterisk.

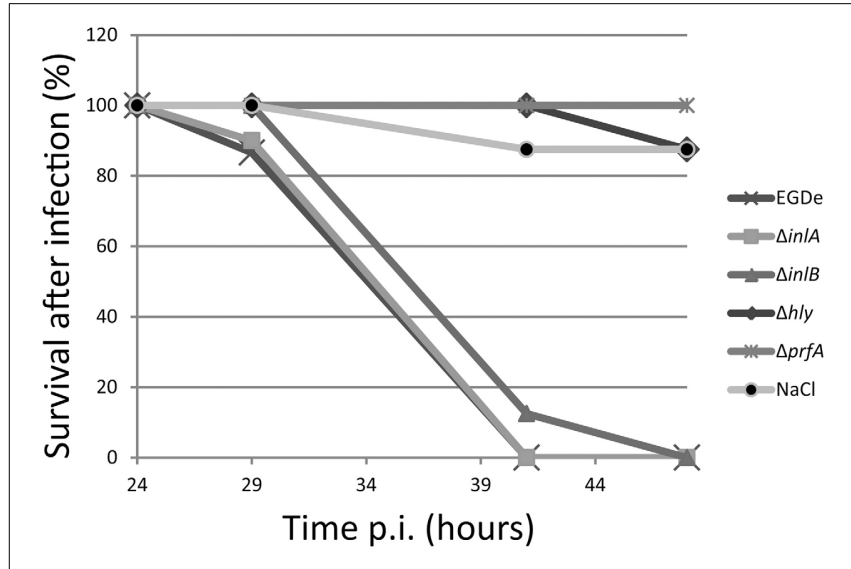

FIGURE 2 | Survival curve of chicken embryos infected with L. monocytogenes wild-type $\Delta$ prfA, $\Delta h / y, \Delta$ inlA or $\Delta$ inlB mutant strains. $\sim 5 \times 10^{2}$ bacteria were injected into 9-day old chicken embryos and death was monitored from 24 to $48 \mathrm{~h}$ post-infection. Death is shown as mean of 2 experiments ( $n=15,9,12,11,12$, and 14 for WT, $\Delta$ prfA, $\Delta h l y$, $\Delta$ inlA, $\Delta$ in/B, and $\mathrm{NaCl}$ respectively).

\section{DISCUSSION}

In this work, we have analyzed if chicken embryos could be used as a model system for studying Listeria monocytogenes pathogenicity. Chicken embryos have previously been used as an infection model system to study the pathogenicity of various bacteria, including L. monocytogenes (Terplan and Steinmeyer, 1989; Buncic and Avery, 1996; Norrung and Andersen, 2000; Olier et al., 2002, 2003; Jiang et al., 2005; Severino et al., 2007; Wang et al., 2008; Horzempa et al., 2010; Yin et al., 2011; Oh et al., 2012; Polakowska et al., 2012; Alnassan et al., 2013). In this study, we have further assessed the chicken embryo as an infection model for Listeria using the well-used L. monocytogenes wild type strain EGDe. By examining isogenic mutant strains, we have also in more depth analyzed several virulence factors controlled by the transcriptional activator PrfA (i.e., LLO, InlA, and InlB). First, we established that the PrfA-pathway was essential to kill chicken embryos (Figure 1A). Also, almost a 100-fold lower number of $\triangle p r f A$ bacteria compared with wild-type bacteria was observed between the strains when analyzing the liver (Figure 1B). When analyzing PrfA-regulated virulence factors, we observed that the pore-forming cytolysin LLO was absolutely required for a successful killing (Figure 2). The finding that both PrfA and LLO are essential for pathogenesis has been shown before (McKay and Lu, 1991; Vazquez-Boland et al., 2001). No one has however, to our knowledge, investigated the roles of the two most important internalins, InlA and InlB, in the chicken embryo model. In contrast to LLO, neither InlA nor InlB were required for a successful Listeria infection of chicken embryos (Figure 2). Similar to humans, the chicken has a proline at position 16 of E-cadherin, allowing a productive interaction between the adhesin InlA and its receptor E-cadherin (Lecuit et al., 1999). This is in contrast to mice which harbor a glutamic acid at position 16 of E-cadherin, preventing a successful interaction and hence invasion. A recent study could give a possible explanation of our results (Roy and Bandyopadhyay, 2014). In that work, the authors showed that the expression of the mRNA encoding Ecadherin was high early during the chicken development in all examined tissues, but diminished in chicken embryos older than 6 days. Since the chicken embryos in this study were 9 days or older, it could be hypothesized that the E-cadherin protein is less abundant and therefore play a less significant role during Listeria infection. The finding that InlB was not important for a chicken embryo infection is less surprising: The c-Met receptor in chicken embryos lacks lysines at position 599 and 600 in the c-Met receptor, which appear essential for a successful InlB:c-Met interaction in humans (Niemann et al., 2007).

Other groups have previously used chicken embryos to examine the virulence potential of clinically and environmentally isolated species of different serotypes by determining mean-time to death (Terplan and Steinmeyer, 1989; Buncic and Avery, 1996; 
Norrung and Andersen, 2000; Olier et al., 2002, 2003; Severino et al., 2007; Yin et al., 2011). We have instead focused this work on a defined bacterial lineage (EGDe). Nevertheless, many results obtained by other groups, using different Listeria lineages, overlap with our study. In particular, the survival curve of the embryo, after inoculation of the wild-type L. monocytogenes strain, was in agreement with previously published work (Buncic and Avery, 1996; Olier et al., 2002, 2003; Severino et al., 2007), indicating that the chicken embryo is a reliable model for studying Listeria infection. The chicken embryo model is well-established for studying development biology (Bénazéraf and Pourquié, 2013), as well as virus infections (Xia et al., 2013) where several tools have been developed. In light of this and through this work, we believe that the chicken embryo model might be a less-expensive but still reliable model for analyzing putative virulence factors compared to the mice model. However, since L. monocytogenes infection of chicken embryos is InlA and InlB independent, we believe that the chicken embryo model does not completely reflect the infection route in humans. It should be noted that L. monocytogenes can cause Listeriosis in avian species, like chickens (Cooper et al., 1992; Vazquez-Boland et al., 2001). The chicken embryo model would therefore be beneficial for the food-industry in order to examine route(s) of infection, but also means to prevent Listeria pathogenesis.

Although gerbils provide functional InlA and InlB pathways, the number of bacteria required to cause still-birth of pregnant gerbils is surprisingly high (Smith et al., 2008). The reason for this is unknown, but it could indicate that certain host factors that are required for an efficient Listeria infection are lacking in gerbils, thus making it a less attractive model. The situation for the chicken model is the opposite: Listeria strains lacking InlA or InlB still are able to kill chicken embryos.

In comparison with mice, the most studied animal model for Listeria infectivity, chickens also have a sequenced genome, are relatively inexpensive and easy to use. Listerial infection of chicken embryos also relies on LLO and PrfA for efficiency. Still, the mice model is preferable since an efficient infection require InlB (and InlA in the transgenic mice, Disson et al., 2008) indicating that this model more closely reflect human listeriosis. Also, the vast amount of mice knock-out mutants will promote the identification of host factors important for Listeria infection.

Despite having a c-Met receptor unable to interact with InlB, the guinea pigs still proves valid as a model organism to study Listeria infection pathways (Williams et al., 2009; Ebersbach et al., 2010; Wu and Matthews, 2013). In analogy to the guinea pig model, we speculate that Listeria uses alternative pathways for entering cells in the chicken embryo. We therefore suggest that the chicken embryo model could be used to identify novel Listeria virulence factors (e.g., other adhesins), that could be masked by an InlA/InlB-dependent mechanism in other animal models.

\section{ACKNOWLEDGMENTS}

Jörgen Johansson was supported by Umeå University, the Swedish Research Council grants K2011-56X-15144-08-6 and 621-20122451 and an ERC starting grant no 260764 - RNAntibiotics. We thank Mr. A. Johansson for technical assistance.

\section{SUPPLEMENTARY MATERIAL}

The Supplementary Material for this article can be found online at: http://www.frontiersin.org/journal/10.3389/fcimb. 2014.00170/abstract

\section{REFERENCES}

Alnassan, A. A., Shehata, A. A., Kotsch, M., Lendner, M., Daugschies, A., and Bangoura, B. (2013). Embryonated chicken eggs as an alternative model for mixed Clostridium perfringens and Eimeria tenella infection in chickens. Parasitol. Res. 112, 2299-2306 doi: 10.1007/s00436-013-3392-5

Bénazéraf, B., and Pourquié, O. (2013). Formation and segmentation of the vertebrate body axis. Annu. Rev. Cell Dev. Biol. 29, 1-26. doi: 10.1146/annurevcellbio-101011-155703

Buncic, S., and Avery, S. M. (1996). Relationship between variations in pathogenicity and lag phase at 37 degrees $\mathrm{C}$ of Listeria monocytogenes previously stored at 4 degrees C. Lett. Appl. Microbiol. 23, 18-22 doi: 10.1111/j.1472765X.1996.tb00020.x

Cabanes, D., Dussurget, O., Dehoux, P., and Cossart, P. (2004). Auto, a surface associated autolysin of Listeria monocytogenes required for entry into eukaryotic cells and virulence. Mol. Microbiol. 51, 1601-1614 doi: 10.1111/j.13652958.2003.03945.x

Cabanes, D., Sousa, S., Cebria, A., Lecuit, M., Garcia-del Portillo, F., and Cossart, P. (2005). Gp96 is a receptor for a novel Listeria monocytogenes virulence factor, Vip, a surface protein. EMBO J. 24, 2827-2838 doi: 10.1038/sj.emboj.7600750

Cooper, G., Charlton, B., Bickford, A., Cardona, C., Barton, J., Channing-Santiago, S., et al. (1992). Listeriosis in California broiler chickens. J. Vet. Diagn. Invest. 4, 343-345 doi: 10.1177/104063879200400322

Cossart, P. (2011). Illuminating the landscape of host-pathogen interactions with the bacterium Listeria monocytogenes. Proc. Natl. Acad. Sci. U.S.A. 108, 19484-19491 doi: 10.1073/pnas.1112371108

de las Heras, A., Cain, R. J., Bieleckal, M. K., and Vazquez-Boland, J. A. (2011). Regulation of Listeria virulence: PrfA master and commander. Curr. Opin. Microbiol. 14, 118-127. doi: 10.1016/j.mib.2011.01.005

Disson, O., Grayo, S., Huillet, E., Nikitas, G., Langa-Vives, F., Dussurget, O., et al. (2008). Conjugated action of two species-specific invasion proteins for fetoplacental listeriosis. Nature 455, 1114-1118 doi: 10.1038/nature07303

Disson, O., and Lecuit, M. (2013). In vitro and in vivo models to study human listeriosis: mind the gap. Microbes Infect. 15, 971-980 doi: 10.1016/j.micinf.2013.09.012

Ebersbach, T., Jorgensen, J. B., Heegaard, P. M., Lahtinen, S. J., Ouwehand, A. C., Poulsen, M., et al. (2010). Certain dietary carbohydrates promote Listeria infection in a guinea pig model, while others prevent it. Int. J. Food Microbiol. 140, 218-224 doi: 10.1016/j.ijfoodmicro.2010.03.030

Freitag, N. E., Port, G. C., and Miner, M. D. (2009). Listeria monocytogenes from saprophyte to intracellular pathogen. Nat. Rev. Microbiol. 7, 623-628. doi: $10.1038 /$ nrmicro2171

Gaillard, J. L., Berche, P., Frehel, C., Gouin, E., and Cossart, P. (1991). Entry of L. monocytogenes into cells is mediated by internalin, a repeat protein reminiscent of surface antigens from gram-positive cocci. Cell 65, 1127-1141 doi: 10.1016/0092-8674(91)90009-N

Gripenland, J., Netterling, S., Loh, E., Tiensuu, T., Toledo-Arana, A., and Johansson, J. (2010). RNAs: regulators of bacterial virulence. Nat. Rev. Microbiol. 8, 857-866 doi: 10.1038/nrmicro2457

Horzempa, J., O’Dee, D. M., Shanks, R. M., and Nau, G. J. (2010). Francisella tularensis DeltapyrF mutants show that replication in nonmacrophages is sufficient for pathogenesis in vivo. Infect. Immun. 78, 2607-2619 doi: 10.1128/IAI.00134-10

Jiang, L. L., Song, H. H., Chen, X. Y., Ke, C. L., Xu, J. J., Chen, N., et al. (2005). Characterization of a mutant Listeria monocytogenes strain expressing green fluorescent protein. Acta Biochim. Biophys. Sin. (Shanghai) 37, 19-24 doi: 10.1093/abbs/37.1.19

Khelef, N., Lecuit, M., Bierne, H., and Cossart, P. (2006). Species specificity of the Listeria monocytogenes InlB protein. Cell. Microbiol. 8, 457-470 doi: 10.1111/j.1462-5822.2005.00634.x

Lecuit, M. (2007). Human listeriosis and animal models. Microbes Infect. 9, 1216-1225 doi: 10.1016/j.micinf.2007.05.009

Lecuit, M., Dramsi, S., Gottardi, C., Fedor-Chaiken, M., Gumbiner, B., and Cossart, P. (1999). A single amino acid in E-cadherin responsible for host specificity 
towards the human pathogen Listeria monocytogenes. EMBO J. 18, 3956-3963 doi: 10.1093/emboj/18.14.3956

Lecuit, M., Vandormael-Pournin, S., Lefort, J., Huerre, M., Gounon, P., Dupuy, C., et al. (2001). A transgenic model for listeriosis: role of internalin in crossing the intestinal barrier. Science 292, 1722-1725 doi: 10.1126/science. 1059852

Levraud, J. P., Disson, O., Kissa, K., Bonne, I., Cossart, P., Herbomel, P., et al. (2009). Real-time observation of listeria monocytogenes-phagocyte interactions in living zebrafish larvae. Infect. Immun. 77, 3651-3660 doi: 10.1128/IAI. 00408-09

Mansfield, B. E., Dionne, M. S., Schneider, D. S., and Freitag, N. E. (2003). Exploration of host-pathogen interactions using Listeria monocytogenes and Drosophila melanogaster. Cell. Microbiol. 5, 901-911 doi: 10.1046/j.14625822.2003.00329.x

McKay, D. B., and Lu, C. Y. (1991). Listeriolysin as a virulence factor in Listeria monocytogenes infection of neonatal mice and murine decidual tissue. Infect. Immun. 59, 4286-4290

Mengaud, J., Ohayon, H., Gounon, P., Mege, R. M., and Cossart, P. (1996). E-cadherin is the receptor for internalin, a surface protein required for entry of L. monocytogenes into epithelial cells. Cell 84, 923-932 doi: 10.1016/S00928674(00)81070-3

Niemann, H. H., Jager, V., Butler, P. J., van den Heuvel, J., Schmidt, S., Ferraris, D., et al. (2007). Structure of the human receptor tyrosine kinase met in complex with the Listeria invasion protein InlB. Cell 130, 235-246 doi: 10.1016/j.cell.2007.05.037

Norrung, B., and Andersen, J. K. (2000). Variations in virulence between different electrophoretic types of Listeria monocytogenes. Lett. Appl. Microbiol. 30, 228-232 doi: 10.1046/j.1472-765x.2000.00704.X

Oh, J. Y., Kang, M. S., Yoon, H., Choi, H. W., An, B. K., Shin, E. G., et al. (2012). The embryo lethality of Escherichia coli isolates and its relationship to the presence of virulence-associated genes. Poult. Sci. 91, 370-375 doi: 10.3382/ps.2011-01807

Olier, M., Pierre, F., Lemaitre, J. P., Divies, C., Rousset, A., and Guzzo, J. (2002). Assessment of the pathogenic potential of two Listeria monocytogenes human faecal carriage isolates. Microbiology 148(Pt 6), 1855-1862.

Olier, M., Pierre, F., Rousseaux, S., Lemaitre, J. P., Rousset, A., Piveteau, P., et al. (2003). Expression of truncated Internalin A is involved in impaired internalization of some Listeria monocytogenes isolates carried asymptomatically by humans. Infect. Immun. 71, 1217-1224 doi: 10.1128/IAI.71.3.1217-1224.2003

Polakowska, K., Lis, M. W., Helbin, W. M., Dubin, G., Dubin, A., Niedziolka, J. W., et al. (2012). The virulence of Staphylococcus aureus correlates with strain genotype in a chicken embryo model but not a nematode model. Microbes Infect. 14, 1352-1362 doi: 10.1016/j.micinf.2012.09.006

Roulo, R. M., Fishburn, J. D., Amosu, M., Etchison, A. R., and Smith, M. A. (2014). Dose Response of Listeria monocytogenes invasion, fetal morbidity, and fetal mortality after oral challenge in pregnant and nonpregnant Mongolian gerbils. Infect. Immun. 82, 4834-4841 doi: 10.1128/IAI.01514-14

Roy, P., and Bandyopadhyay, A. (2014). Spatio-temporally restricted expression of cell adhesion molecules during chicken embryonic development. PLoS ONE 9:e96837 doi: 10.1371/journal.pone.0096837

Severino, P., Dussurget, O., Vencio, R. Z., Dumas, E., Garrido, P., Padilla, G., et al. (2007). Comparative transcriptome analysis of Listeria monocytogenes strains of the two major lineages reveals differences in virulence, cell wall, and stress response. Appl. Environ. Microbiol. 73, 6078-6088 doi: 10.1128/AEM.02730-06

Shen, Y., Naujokas, M., Park, M., and Ireton, K. (2000). InIB-dependent internalization of Listeria is mediated by the Met receptor tyrosine kinase. Cell 103 501-510 doi: 10.1016/S0092-8674(00)00141-0
Smith, M. A., Takeuchi, K., Anderson, G., Ware, G. O., McClure, H. M., Raybourne, R. B., et al. (2008). Dose-response model for Listeria monocytogenes-induced stillbirths in nonhuman primates. Infect. Immun. 76, 726-731 doi: 10.1128/IAI.01366-06

Terplan, G., and Steinmeyer, S. (1989). Investigations on the pathogenicity of Listeria spp. by experimental infection of the chick embryo. Int. J. Food Microbiol. 8, 277-280 doi: 10.1016/0168-1605(89)90027-5

Thomsen, L. E., Slutz, S. S., Tan, M. W., and Ingmer, H. (2006). Caenorhabditis elegans is a model host for Listeria monocytogenes. Appl. Environ. Microbiol. 72, 1700-1701 doi: 10.1128/AEM.72.2.1700-1701.2006

Vazquez-Boland, J. A., Kuhn, M., Berche, P., Chakraborty, T., Dominguez-Bernal, G., Goebel, W., et al. (2001). Listeria pathogenesis and molecular virulence determinants. Clin. Microbiol. Rev. 14, 584-640 doi: 10.1128/CMR.14.3.584640.2001

Wang, X., Carmichael, D. W., Cady, E. B., Gearing, O., Bainbridge, A., Ordidge, R. J., et al. (2008). Greater hypoxia-induced cell death in prenatal brain after bacterial-endotoxin pretreatment is not because of enhanced cerebral energy depletion: a chicken embryo model of the intrapartum response to hypoxia and infection. J. Cereb. Blood Flow Metab. 28, 948-960 doi: 10.1038/sj.jcbfm. 9600586

Williams, D., Castleman, J., Lee, C. C., Mote, B., and Smith, M. A. (2009). Risk of fetal mortality after exposure to Listeria monocytogenes based on dose-response data from pregnant guinea pigs and primates. Risk Anal. 29, 1495-1505 doi: 10.1111/j.1539-6924.2009.01308.x

Wollert, T., Pasche, B., Rochon, M., Deppenmeier, S., van den Heuvel, J., Gruber, A. D., et al. (2007). Extending the host range of Listeria monocytogenes by rational protein design. Cell 129, 891-902 doi: 10.1016/j.cell.2007.03.049

Wu, W. H., and Matthews, K. R. (2013). Susceptibility of aged guinea pigs to repeated daily challenge with Listeria monocytogenes. Foodborne Pathog. Dis. 10, 284-289 doi: 10.1089/fpd.2012.1292

Xia, H., Zhao, J., Li, Y., Yin, S., Tang, S., Zhang, Z., et al. (2013). Infection and propagation of Crimean-Congo hemorrhagic fever virus in embryonated chicken eggs. Virus Res. 173, 344-349 doi: 10.1016/j.virusres.2013.01.008

Yin, Y., Tian, D., Jiao, H., Zhang, C., Pan, Z., Zhang, X., et al. (2011). Pathogenicity and immunogenicity of a mutant strain of Listeria monocytogenes in the chicken infection model. Clin. Vaccine Immunol. 18, 500-505 doi: 10.1128/CVI. 00445-10

Conflict of Interest Statement: The authors declare that the research was conducted in the absence of any commercial or financial relationships that could be construed as a potential conflict of interest.

Received: 18 October 2013; accepted: 17 November 2014; published online: 10 December 2014.

Citation: Gripenland J, Andersson C and Johansson J (2014) Exploring the chicken embryo as a possible model for studying Listeria monocytogenes pathogenicity. Front. Cell. Infect. Microbiol. 4:170. doi: 10.3389/fcimb.2014.00170

This article was submitted to the journal Frontiers in Cellular and Infection Microbiology.

Copyright (c) 2014 Gripenland, Andersson and Johansson. This is an open-access article distributed under the terms of the Creative Commons Attribution License (CC BY). The use, distribution or reproduction in other forums is permitted, provided the original author(s) or licensor are credited and that the original publication in this journal is cited, in accordance with accepted academic practice. No use, distribution or reproduction is permitted which does not comply with these terms. 